\title{
Experiencia piloto con el uso extendido de un anticonceptivo oral combinado de baja dosis de estrógeno
}

\author{
Ernesto Pizarro O. \\ Director, Sociedad Chilena de Endocrinología Ginecológica.
}

\section{RESUMEN}

Objetivo: Evaluar en una experiencia piloto la aceptabilidad y bienestar de la usuaria, después de un periodo de 12 meses de un anticonceptivo hormonal combinado oral de baja dosis estrogénica, con modalidad de uso extendido de 84 píldoras activas consecutivas, seguidas de 7 días de placebo. Método: Se incorporan 25 mujeres voluntarias que usan una combinación de 20 ug de etinilestradiol $+3 \mathrm{mg}$ de drospirenona, con un tiempo de uso de 12 meses. En calendario de registro diario se consignan los días de sangrado o goteo genital así como todo tipo de fármaco ingerido. Al término de los 12 meses se efectúa una encuesta respecto al grado de satisfacción con la posología recibida. Resultados: 13 usuarias (52\%) cumplen los 12 meses de uso. Todas ellas manifiestan un alto grado de conformidad, destacando las ventajas de presentar periodos menstruales ocasionales, mejoría marcada en la sintomatología compatible con el síndrome de tensión premenstrual con el consiguiente incremento del bienestar general. Siete usuarias (28\%) no terminan el estudio por razones médicas, siendo 6 de estas por alteraciones de los flujos rojos y en 5 casos (20\%) se producen retiros no médicos. Conclusión: Esta experiencia, que es la primera con la formulación descrita, confirma en un porcentaje de usuarias las bondades adicionales reportadas en las experiencias previas con otros productos similares, respecto al uso extendido de anticoncepción hormonal oral en un grupo de mujeres que deseaban espaciar sus periodos menstruales.

\section{PALABRAS CLAVE: Anticoncepción hormonal de uso extendido, drospirenona, síndrome tensión premenstrual}

\section{SUMMARY}

Objetives: A pilot study designed in order to know about the acceptability of an oral combined contraceptive pill, with low estrogen dose used in an extended way of 84 consecutive days, followed by 7 days of no pills ingestion during a scheduled follow up of 1 year. Methods: 25 volunteers women were recruited among those which wants to use oral combined contraceptive pills and who accepted this extended way of use. Combined contraceptive pills contains each $20 \mathrm{ug}$ of ethinylestradiol plus $3 \mathrm{mg}$ of drosperinone. At admission women were provided with menstrual diary cards in order to check all bleeding days plus any extra pharmaceutical compound received. At the end of the 12 months follow up an interview was done in order to know women experiences and acceptability of this extended way of use. Results: $52 \%$ of women end the study at the scheduled 12 months use. All of them feel that the main advantages of these extended way of use were to have few menstrual periods and improving premenstrual symtomatology with better quality of life. 7 women (28\%) did not finish the study because of medical reasons being due in 6 of these for bleeding disturbances. Conclusions: This report is pioneer with the use this hormone combination in this extended 
type way of use. Results confirm previous positive reports experiences using another similar hormonal compounds with this extended way of use in women who want to reduce the interval of their menstrual periods.

\section{KEY WORDS: Oral combined contraception, drospirenone, premenstrual syndrome}

\section{INTRODUCCIÓN}

La aparición en los años sesenta de la anticoncepción hormonal, así como la de la contracepción intrauterina, trae como consecuencia en los años siguientes, cambios de actitudes tanto en la pareja así como en la mujer misma con repercusiones en aspectos sociales, demográficos, económicos y sanitarios. La inserción progresiva de la mujer en los campos educacionales, sociales y laborales, tanto en nuestro país como en el mundo entero, ha creado la necesidad de paliar o perfeccionar desmedros biológicos comparativos de la mujer respecto al hombre, como lo son entre otros, los derivados de su ciclicidad menstrual, la que aparte de su desmedro sanitario. Implica, en algunos casos, amenazas periódicas en su estado de salud tanto física o mental, como lo son las condiciones clínicas de hipermenorrea, anemia secundaria, dismenorrea y perturbaciones en la esferas anímicas o psicológicas derivadas del llamado síndrome de tensión premenstrual, presente según el Consenso de Expertos de Latinoamérica entre el $48 \%$ al $90 \%$ de las mujeres y a su expresión más severa, denominada síndrome disfórico premenstrual, con prevalencias entre el 2 al 9\% (1). En estas entidades clínicas se destacan cambios cíclicos en el temperamento de la mujer como son la labilidad emocional, con mayor sensibilidad y susceptibilidad, irritabilidad, angustia, ansiedad, y en los casos más extremos, como lo es en el síndrome disfórico premenstrual, depresiones prolongadas o transitorias. Aparte, se agregan retenciones hídricas efímeras con aumentos ocasionales de peso en el periodo premenstrual. Se ha logrado paliar parcialmente estos síntomas con la aparición de la anticoncepción oral combinada $(A O C)$ de uso intermitente con periodos de descanso transitorios de 4 o 7 días después de 21 o 24 días de uso con el fin de homologar artificialmente el ciclo menstrual natural de la mujer. Con el dirigido propósito de espaciar, y por tanto minimizar todos los efectos deletéreos descritos derivados de la mensual periodicidad del ciclo menstrual en el año 2003, el Food Drug Administration (FDA) de los EEUU de Norteamérica aprobó después de una gran y acuciosa experiencia, un sistema de uso extendido de $A O C$, en base a etinil estradiol (EE) 30 ug y levonorgestrel (LNG) 150 ug, con 84 días de uso continuo del anticonceptivo y con intervalos de 7 días de descanso, con el preciso objetivo de disminuir de 13 a 4 los periodos menstruales en el año (2). Comunicaciones avalan los deseos de poblaciones de mujeres en espaciar sus periodos menstruales, considerando algunas de ellas el tener periodos menstruales cada 3 meses como el ideal (3) y en otras el disminuir los síntomas derivados de la menstruación misma (4). El $60 \%$ de las mujeres italianas prefieren tener sangrados menos de una vez al mes, no necesariamente por razones médicas, sino por conveniencias prácticas, lo cual concuerda con datos provenientes de otros países europeos (5). Respondiendo a la creciente aceptabilidad por parte de determinadas usuarias de este uso extendido de AOC siguen apareciendo publicaciones con el uso de esta forma de anticoncepción, usando ya sea distintas fórmulas hormonales combinadas orales, considerando aspectos tanto clínicos $(6,7,8,9)$ como variaciones metabólicas $(10,11)$ o en la forma de anillos vaginales $(12,13)$.

Con el objetivo de evaluar la aceptabilidad clínica con una formula de uso extendido es que planteamos esta experiencia piloto usando un componente combinado hormonal oral con una baja dosis estrogénica (EE 20 ug) y con un progestágeno de cuarta generación, como es la drospirenona (DRSP) $3 \mathrm{mg}$, con reconocidos efectos antiandrogénicos y antimineralocorticoídeos.

\section{PACIENTES Y MÉTODO}

Durante un periodo de 8 meses se enrolaron un total de 25 mujeres voluntarias, atendidas en la consulta privada, que tenían indicación médica de anticoncepción hormonal combinada oral, que manifestaron su deseo de usar esta en forma continua durante 84 días, con periodos subsecuentes de 7 días de descanso, durante un año. Después de haber recibido explicaciones y detalles de la naturaleza de esta experiencia piloto, dieron su consentimiento verbal para su participación en esta observación. El anticonceptivo usado fue una combinación monofásica de DRSP $3 \mathrm{mg}+20$ ug de EE en forma de píldoras que fueron provistas gratuitamente a las usuarias. 
Los criterios de reclutamiento fueron: voluntarias de 18 o más años, que entendieran la naturaleza del estudio, que inicialmente estuviesen dispuestas a usar el anticonceptivo por un periodo de 1 año, que fueran o no usuarias de otras formas de píldoras anticonceptivas combinadas al momento de la admisión, que no tuvieran ninguna contraindicación para el uso de AOC y que estuvieran dispuestas a llenar un calendario menstrual en el cual se consignaban los días de sangrado o goteo menstrual y la ingesta adicional de cualquier tipo de medicamentos. Los criterios de exclusión incluyeron: amenorrea; anomalías en el examen ginecológico del aparato reproductor incompatibles con el uso de anticoncepción combinada; cualquier contraindicación absoluta o relativa para el uso de la misma, tanto en la anamnesis como en el examen médico general, de acuerdo a los criterios de elegibilidad del uso de anticoncepción hormonal, establecidas en las guías de la Organización Mundial de la Salud (14).

Se planificaron controles de seguimiento cada 3 meses, momento en el cual se preguntó por el bienestar de cada usuaria, se revisó el calendario menstrual y se proveyó a cada una de ellas con las píldoras anticonceptivas por los 3 meses siguientes, y se efectuó, al igual que al inicio del estudio, un control de peso, presión arterial y examen ginecológico correspondiente.

Las participantes tuvieron la posibilidad de efectuar visitas no programadas en cualquier momento que ellas estimaran, al igual que la opción de un contacto telefónico permanente. En el control final, además se efectuó una breve encuesta respecto a la experiencia y aceptabilidad con esta modalidad anticonceptiva, y repercusiones de esta sobre el perfil de sangrado menstrual, así como sobre la esfera física o psíquica de la usuaria.

\section{RESULTADOS}

De las 25 usuarias aceptadas, 13 (52\%) terminan el estudio y $12(48 \%)$ no lo finalizan. De estas últimas, 7 son retiros médicos $(28 \%)$ y 5 son retiros no médicos $(20 \%)$. La edad promedio de todas las usuarias fue de 31,5 años.

En la Tabla I se presenta las características generales de edad, paridad y uso previo de anticoncepción hormonal de las 13 mujeres que terminaron el estudio. La edad promedio fue de 32,2 años (rango: $19-48$ años). El 53,9\% (7/13) de ellas no tenía paridad previa. El 15,4\% (2/13) no habían usado anticoncepción hormonal anteriormente.
En la Tabla Il se presenta las características generales de edad, paridad, uso previo de anticoncepción hormonal de las usuarias que fueron retiradas por motivos médicos. La edad promedio fue 30,7 años (rango: 23-43 años). El 85,7\% (6/7) de ellas no tenía paridad previa. El $28,6 \%(2 / 7)$ no habían usado anticoncepción hormonal anteriormente. El 85,7\% (6/7) suspendió por goteo persistente.

En la Tabla III se presenta las características generales de edad, paridad, uso previo anticoncepción hormonal de las usuarias que fueron retiradas por motivos no médicos. La edad promedio fue 30,8 años (rango: $22-41$ años). El 60\% (3/5) no tenía paridad previa. Todas habían usado anticoncepción hormonal anteriormente. Los motivos de retiro fueron diversos, solo una de ellas por deseo de embarazo.

En la Tabla IV se presenta la presión arterial y peso de las usuarias que terminaron el estudio, al ingreso y término del estudio. No se aprecian cambios significativos de los valores promedios de las variables evaluadas.

En la Tabla $V$ se presenta el grado de satisfacción por la forma extendida de anticoncepción en las usuarias que terminaron el estudio. Prácticamente todas presentaron un grado de satisfacción máxima con esta forma de anticoncepción.

Tabla I

CARACTERÍSTICAS DE LAS USUARIAS QUE TERMINARON EL ESTUDIO

\begin{tabular}{cccc}
\hline Usuaria & Edad & Paridad & $\begin{array}{c}\text { Uso previo de AC } \\
\text { hormonal }\end{array}$ \\
\hline 1. ML & 43 & 0 & No \\
2. EL & 48 & 2 & ACO \\
3. CG & 32 & 2 & ACO \\
4. MO & 23 & 0 & ACO \\
5. AL & 32 & 2 & ACO \\
6. MZ & 45 & 1 & ACO \\
7. MR & 29 & 0 & ACO \\
8. PY & 20 & 0 & ACO \\
9. CM & 19 & 0 & No \\
10. CD & 24 & 0 & ACO \\
11. NR & 20 & 0 & ACO \\
12. MS & 42 & 2 & Progestágeno solo \\
13. AV & 42 & 3 & ACO \\
\hline
\end{tabular}

AC: anticoncepción. ACO: anticonceptivos orales. 
Tabla II

\section{CARACTERÍSTICAS DE LAS MUJERES QUE SE RETIRARON DEL ESTUDIO POR MOTIVOS MÉDICOS}

\begin{tabular}{lcccc}
\hline Usuaria & Edad & Paridad & $\begin{array}{c}\text { Uso previo de } \\
\text { AC hormonal }\end{array}$ & $\begin{array}{c}\text { Motivo } \\
\text { de retiro }\end{array}$ \\
\hline 1. XC & 38 & 0 & ACO & Mioma \\
2. CR & 23 & 0 & ACO & Goteo \\
3. RU & 24 & 0 & ACO & Goteo \\
4. PL & 30 & 0 & ACO & Goteo \\
5. FT & 30 & 0 & No & Goteo \\
6. FC & 27 & 0 & ACO & Goteo \\
7. CR & 43 & 3 & No & Goteo \\
\hline
\end{tabular}

AC: anticoncepción. ACO: anticonceptivos orales.

Tabla III

CARACTERÍSTICAS DE LAS MUJERES QUE SE RETIRARON DEL ESTUDIO POR MOTIVOS NO MÉDICOS

\begin{tabular}{|c|c|c|c|c|}
\hline Usuaria & Edad & Paridad & $\begin{array}{l}\text { Uso } \\
\text { previo de } \\
\text { ACO }\end{array}$ & $\begin{array}{l}\text { Motivo de } \\
\quad \text { retiro }\end{array}$ \\
\hline 1. JG & 33 & 0 & $\mathrm{Si}$ & $\begin{array}{l}\text { Deseo de } \\
\text { embarazo }\end{array}$ \\
\hline 2. MV & 41 & 2 & $\mathrm{Si}$ & $\begin{array}{l}\text { Dejó de } \\
\text { necesitar } \\
\text { anticoncep- } \\
\text { ción }\end{array}$ \\
\hline 3. JJ & 35 & 2 & $\mathrm{Si}$ & Olvido \\
\hline 4. $C L$ & 23 & 0 & $\mathrm{Si}$ & $\begin{array}{l}\text { Sin se- } \\
\text { guimiento } \\
\text { (extranjero) }\end{array}$ \\
\hline 5. NM & 22 & 0 & $\mathrm{Si}$ & $\begin{array}{l}\text { Abandona } \\
\text { por temor a } \\
\text { amenorrea }\end{array}$ \\
\hline
\end{tabular}

ACO: anticonceptivos orales.

\section{DISCUSIÓN}

Los estudios pilotos constituyen una útil herramienta para la indagación inicial respecto a dimensionar la real necesidad en efectuar una futura investigación científica, con casuísticas adecuadas para resolver hipótesis aun no resueltas. En esta pequeña casuística hemos privilegiado el uso de una combinación anticonceptiva de amplia aceptabilidad en el momento actual por contener una baja dosis estrogénica (EE 20 ug/dia), la cual ha evidenciado menores incidencias de efectos secundarios derivados
Tabla IV

PRESIÓN ARTERIAL Y PESO DE LAS MUJERES AL INICIO Y TÉRMINO DEL ESTUDIO

\begin{tabular}{lcccc}
\hline & \multicolumn{2}{c}{ Presión arterial $(\mathrm{mmHg})$} & \multicolumn{2}{c}{ Peso $(\mathrm{kg})$} \\
Usuaria & Inicial & Final & Inicial & Final \\
\hline 1. ML & $125 / 80$ & $100 / 70$ & 69,1 & 69,2 \\
2. EL & $120 / 80$ & $110 / 60$ & 62,4 & 64,0 \\
3. CG & $110 / 70$ & $110 / 80$ & 57,6 & 57,8 \\
4. MO & $120 / 75$ & $125 / 80$ & 54,0 & 53,9 \\
5. AL & $120 / 70$ & $120 / 80$ & 52,5 & 55,9 \\
6. MZ & $105 / 80$ & $90 / 60$ & 63,2 & 65,4 \\
7. MR & $120 / 70$ & $130 / 75$ & 58,6 & 55,0 \\
8. PY & $100 / 65$ & $110 / 60$ & 51,8 & 53,5 \\
9. CM & $120 / 60$ & $110 / 70$ & 47,5 & 47,3 \\
10. CD & $125 / 80$ & $120 / 70$ & 59,2 & 61,0 \\
11. NR & $135 / 75$ & $130 / 80$ & 71,9 & 72,4 \\
12. MS & $110 / 60$ & $100 / 60$ & 51,5 & 50,4 \\
13. AV & $130 / 85$ & $120 / 70$ & 57,5 & 56,5 \\
Promedio & $118 / 73$ & $113 / 70$ & 58,2 & 58,6 \\
\hline
\end{tabular}

\section{Tabla V}

SATISFACCIÓN DE USUARIA QUE TERMINARON EL ESTUDIO CON EL USO DE ANTICONCEPCIÓN EXTENDIDA

\begin{tabular}{lcccc}
\hline Usuaria & $\begin{array}{c}\text { Agrado } \\
\text { con el } \\
\text { sistema }\end{array}$ & $\begin{array}{c}\text { Lo conti- } \\
\text { nuaría }\end{array}$ & $\begin{array}{c}\text { Lo reco- } \\
\text { mendaría }\end{array}$ & $\begin{array}{c}\text { Aspectos } \\
\text { negativos }\end{array}$ \\
\hline 1. ML & 10 & 10 & 10 & No \\
2. EL & 10 & 10 & 10 & No \\
3. CG & 10 & 10 & 10 & No \\
4. MO & 8 & 6 & 10 & $\begin{array}{c}\text { Goteo } \\
\text { inicial }\end{array}$ \\
5. AL & 10 & 10 & 10 & No \\
6. MZ & 10 & No* & 10 & No \\
7. MR & 10 & 10 & 10 & No \\
8. PY & 10 & 10 & 10 & No \\
9. CM & 8 & 10 & 9 & Goteo \\
& 10 & 10 & 10 & ocasional \\
10. CD & 10 & & Goteo \\
& 10 & 10 & 10 & inicial \\
11. NR & 9 & 10 & 10 & * * \\
12. MS & 10 & 10 & 10 & No \\
13. AV & 10 & & & No
\end{tabular}

* dice que recibió mail diciendo que la ausencia de menstruaciones favorecía tumor cerebral. ${ }^{\star \star}$ goteo al ingerir simultáneamente otros fármacos. Escala 1 - 10, siendo: $1=$ mínimo, 10= máximo. 
de los estrógenos y una menor incidencia de complicaciones mayores, como es el tromboembolismo venoso y un progestágeno de última generación con propiedades antiandrogénicas y antimineralocorticoidea, como lo es la DRSP (15). Clínicamente, esta última cualidad se traduce en una menor retención hídrica, con la eventual ventaja de una mínima variación en el peso corporal y ser considerada esta como un factor desencadenante del llamado síndrome de tensión premenstrual. Al respecto, en los últimos años la Food Drug Administration (FDA), aprobó esta forma anticonceptiva combinada de DRSP $3 \mathrm{mg}+\mathrm{EE} 20 \mathrm{ug}$ con un régimen de ingesta 24/4, para el manejo del acné moderado y el tratamiento de los síntomas emocionales y físicos del síndrome disfórico premenstrual $(16,17)$.

Recientes publicaciones confirman las bajas tasas de embarazo con esta formulación, con índice de Pearl corregido de 0,22 embarazos en 11.755 ciclos colectados en 50 centros europeos (18), al igual que aceptables perfiles de sangrado y control de ciclo, comparable al de otros AOC ya establecidos (19).

Actualmente, el uso de la forma extendida de anticoncepción hormonal combinada ha sido motivo de especial preocupación científica, como lo avalan las recientes publicaciones detallando tanto las distintas experiencias clínicas con diferentes formulas $(6,7,8,9,20)$, así como las consecuencias metabólicas en las usuarias después de 12 meses de uso $(10,11)$. Innegables aparecen las ventajas de esta forma especial de uso de anticoncepción hormonal, en los grupos de usuarias afectas al denominado síndrome de tensión y/o disfórico premenstrual, así como en todas aquellas con el sostenido deseo de espaciar sus periodos menstruales.

Un objetivo adicional de esta publicación es revisar y traspasar las inquietudes científicas y comunicaciones respecto a esta forma especial de uso de anticoncepción combinada hormonal. En la pionera publicación de Anderson y cols (2), en una experiencia comparativa randomisada, multicéntrica, con 1 año de seguimiento, efectuada en 47 centros de EEUU y con un total de 682 mujeres norteamericanas incorporadas, en la que se compara el uso de una forma de $A O C$ de uso tradicional (21/7) que contiene 30 ug de EE + 150 de LNG versus una forma extendida del mismo producto, con 84 días de uso consecutivo de píldoras activas seguidas por 7 días de placebo, se muestra que esta última formulación es efectiva, inocua y bien tolerada. Inicialmente, se reportan más visitas no programadas por sangrados intermenstruales con el producto de uso extendido, pero estas disminuyen progresivamente equiparándose después del cuarto mes de uso entre ambos grupos. Al término del estudio, la mayoría de las usuarias manifiesta su satisfacción con el uso extendido del AOC, catalogándolo como bueno o excelente y recalcan el gran agrado que les produjo el tener menos periodos menstruales (2). Posteriormente, el mismo autor comunica los resultados de estudios histológicos endometriales efectuados en 50 usuarias de la forma extendida después de 1 año de uso, reportando que no se registran casos de hiperplasias $u$ otras patologías endometriales (21).

Paralelamente, en Europa se suceden comunicaciones respecto al uso de anticoncepción hormonal de uso extendido. En el VIII Congreso de la Sociedad Europea de Contracepción, Mueck y cols (22), comparan el uso de una fórmula de uso extendido (42-126 días) versus una tradicional 217, usando DRSP 3mg + EE 30ug/día. Concluyen, que los síntomas de alivio premenstrual y el mejor bienestar, se acrecientan a medida que se prolonga el uso del preparado extendido, y plantean que la sintomatología de mastalgia, edema y reducción en los problemas de la piel deben ser mejorados con este sistema especial de ingesta del AOC. Enfatizan además, que el alivio de la dismenorrea y el menor sangrado menstrual son considerados como muy positivos por la mayoría de las mujeres al término de la observación.

Machado y cols (23), presentan una experiencia con el fin de analizar el perfil de sangrado en 32 mujeres brasileñas con el uso consecutivo durante 6 meses de una fórmula conteniendo 75 ug de gestodeno +30 ug de EE. Concluyen que esta formulación presenta un aceptable perfil de sangrado consistente primordialmente en goteo o "spotting". La incidencia de amenorrea fue alta después del cuarto mes de uso.

El mismo grupo (24), muestra una casuística de uso extendido por 56 días con igual formulación farmacológica en 334 mujeres, que eran usuarias inicialmente de ingesta de la forma clásica de 21 + 7 días de descanso, de diferentes tipos de AOC. Previo a la experiencia, el $65 \%$ de las 334 usuarias presentaba alguna sintomatología en el intervalo libre del uso del AOC. Al final la experiencia mostró que los efectos colaterales de dolor de cabeza, mastalgia, dismenorrea, edema, menorragia y labilidad emocional disminuyen significativamente después de los 56 días de uso continuo del AOC, no observando disminución significativa en los síntomas de náuseas, depresión, aumento de peso o reducción de la libido.

En el $X$ Congreso de la Sociedad Europea de Contracepción, numerosas comunicaciones se presentaron relacionadas con el uso extendido de los AOC. Entre otras, Seidman y cols (25) reportan en 
109 mujeres de Israel que el uso extendido por 84 días de una fórmula de 30 ug de $\mathrm{EE}+3 \mathrm{mg}$ de DRSP fue altamente aceptable, sin aumento de efectos colaterales, y con una disminución de los días de sangrado. Prilepskaya y cols (26), en 112 mujeres rusas demuestra mejores efectos sobre la dismenorrea y el síndrome de tensión premenstrual al usar una fórmula de 30ug de EE + 150ug de desogestrel con 63 píldoras activas consecutivas, seguidas de 7 días de placebo durante 1 año en comparación al uso en la forma cíclica tradicional del mismo producto. Mirjana y cols (27), presentan un estudio demostrando la alta aceptabilidad de mujeres serbias con el uso extendido por 24 semanas consecutivas de una formulación 30 ug de EE + dienogest con fines terapéuticos, pero no así con fines anticonceptivos. Barreiros y cols (28), comparan los resultados sobre el perfil menstrual en 150 mujeres brasileñas usando un anillo vaginal que libera 120 ug/dia de etonogestrel + EE 15 ug/día, versus un producto oral de 150 ug de desogestrel + 30 ug EE con uso extendido de ambos por 81 días con 7 días de descanso durante 1 año; demuestran que a medida que transcurre el tiempo disminuyen con ambos productos los días de goteo o sangrado intermenstrual, siendo esta disminución más evidente con el anillo vaginal. Plantean por tanto nuevas perspectivas en la forma de uso de este anillo. Guazelli y cols (29), comparan diferentes parámetros a un año plazo usando el anillo vaginal con un AOC de 30 ug de EE + 75 ug de gestodeno en la forma de ciclo extendido de 84 -7 días.

Recientes comunicaciones continúan evaluando las distintas formas de anticoncepción hormonal de uso extendido. Teichmann y cols (8), en un estudio multicéntrico randomisado colaborativo entre 44 centros europeos, presentan resultados después de 1 año de uso de una fórmula de uso extendido permanente de LNG 90 ug/día + EE 20 ug/día, versus la forma cíclica de 21 días de 100 ug de LNG +20 ug de EE. Concluyen diciendo que la forma continua es inocua y efectiva. Recalcan la ventaja de esta última al no requerir protección sanitaria adicional.

Kroll y cols (9), presentan resultados en 1249 mujeres norteamericanas usando durante un año ciclos extendidos de 84 días con una combinación de 100 ug de LVN + 20 ug de EE, adicionando 7 días de EE, en vez del tradicional placebo; concluyen informando que esta formulación muestra una efectiva protección del embarazo, es bien tolerada y presenta efectos adversos similares a las otras formulaciones de bajas dosis anteriormente reportadas (7).

Bonassi Machado y cols (6), randomizan y comparan los efectos en 78 mujeres usando, por un periodo extendido continuo de 168 días, una formula- ción ya sea de 30 ug de EE + 3 mg de DRSP o de la misma formulación usada durante el mismo tiempo en forma cíclica tradicional de 21 días +7 días de intervalo libre de hormona; terminan comunicando que en el grupo continuo se mejoran significativamente las condiciones de dismenorrea, dolor de cabeza, acné, náuseas, e incremento del apetito y que los síntomas de mastalgia y edema lo hacen en el grupo cíclico.

Guazzelli y cols (12), reportan un estudio comparativo entre el anillo vaginal con liberación de 120 ug de etonogestrel y 15 ug de EE/ día y la forma oral de 30 ug de EE +150 ug de desogestrel usados ambos en ciclo extendido de 84 días con 7 días de descanso por el periodo de 1 año. Los días de sangrado o goteo menstrual disminuyen significativamente con ambos componentes, siendo esta reducción significativamente menor en el grupo oral; durante este periodo hay una significativa reducción con ambos productos en el goteo o sangrado intermenstrual siendo esta significativamente menor en las usuarias del anillo vaginal. En este mismo grupo de 75 mujeres, Barreiros y cols (13), comunican sintomatología en las usuarias del anillo vaginal antes del inicio y después del año de uso de este con el sistema extendido ya descrito. En comparación a las condiciones previas al uso, al término de la observación las usuarias presentan significativamente menos dismenorrea e irritabilidad, y una reducción no significativa en mastalgia, dolor en extremidades inferiores y cefalea. No se registran cambios en la presión arterial. Aunque existe un significativo aumento en el peso y en el índice de masa corporal, estos permanecen dentro de los márgenes de la variación biológica esperada.

Wiegratz y cols (11), demuestran que después de 12 meses de uso al comparar una formulación extendida de $84+7$ días de intervalo, con 30 ug EE + $2 \mathrm{mg}$ dienogest con el uso cíclico convencional de estos mismos componentes, no hay diferencias en ninguno de los parámetros del metabolismo lipídi$\mathrm{co}$, siendo estos por el contrario muy comparables.

Bonassi y cols (10), igualmente muestran los impactos metabólicos sobre los parámetros lipídi$\cos$, de carbohidratos y de coagulación después de uso continuado por 168 días versus el uso tradicional de 21 días +7 días de descanso, por 6 ciclos, de una formulación de 30 ug de EE $+3 \mathrm{mg}$ DRSP. En ambos grupos las alteraciones son similares.

Si bien en nuestra experiencia piloto la muestra es pequeña algunos comentarios podemos hacer al respecto. El $52 \%$ de la población estudiada termina el estudio, en el lapso previamente programado de 12 meses de uso, cifra muy semejante a lo que ocurre en grupos poblacionales usuarias de $A O C$ 
con el tradicional sistema de uso con descansos intermitentes mensuales. En una reciente publicación (6), se demuestran tasas de abandono semejantes $(25,6 \%$ y $23 \%)$ al usar en forma continua o con intervalos 21/7 durante 6 meses una combinación de AOC de 30 ug de EE + 3 mg DRSP. Si corregimos eliminando a las 5 usuarias que terminan el estudio por razones no médicas, resulta que las 13 pacientes que terminan satisfactoriamente corresponden al $65 \%$ del total de las 20 mujeres que pueden ser realmente observadas.

En nuestros resultados, prácticamente la totalidad de las mujeres que aceptó el uso de esta forma extendida y que termina el estudio, lo hace en forma muy satisfactoria y desea seguir con el uso de esta modalidad mas allá de los 12 meses de uso inicialmente programados. Además, manifiestan sentirse muy dispuestas a recomendar esta forma de ingesta a otras usuarias, destacando entre sus virtudes la menor cantidad de menstruaciones, la ausencia y/o mejoría del síndrome de tensión premenstrual, la casi nula retención hídrica y la ausencia de perturbaciones significativas de su estado de bienestar general. No se registraron embarazos en el total de meses observados. Los resultados aquí obtenidos, sumados a los datos de la literatura actual, nos hacen reflexionar en que esta modalidad extendida del uso de AOC es una alternativa más que debiera ser planteada u ofrecida a toda mujer con indicación médica de anticoncepción hormonal y que su uso, es una permisible opción en toda mujer que lo requiera y así lo decida.

Los estudios recientes de Hannaford y cols (30), respecto al no incremento de la mortalidad al comparar 46.000 mujeres que toman o han tomado $A O C$ versus las que nunca lo han hecho, en un estudio de cohorte después de 38 años de observación en el Reino Unido, constituyen un aval en el uso, ya sea de ésta modalidad extendida de ingesta de AOC o en el uso cíclico tradicional de la misma. Esta situación es de especial importancia en aquellos grupos sanitarios que hemos vivido paralela y cercanamente al desarrollo y evolución de la anticoncepción hormonal y las catastróficas consecuencias en la salud pública e individual derivadas del aborto, legal o ilegal, y de los embarazos no programados o no deseados. La adicional publicación de Hannaford y cols (31), respecto al no incremento de cánceres en general en usuarias de AOC, y la reciente comunicación de Vessey y cols (32), que reportan riesgos relativos de mortalidad en más de 17.000 usuarias de AOC versus nunca usuarias, después de un seguimiento de aproximadamente 40 años (Ca de mama RR: 1,0; IC: 0,8-1,2; Ca uterino, exceptuando el de cérvix, RR: 0,3; IC: 0,1-0,8;
Ca de ovario RR: 0,4; IC: 0,3-0,6), reportando que la protección de este tipo de cánceres aumenta con la duración del uso del AOC y que persiste por más de 20 años después de la supresión del mismo; encontrando además que la mortalidad por enfermedades del aparato circulatorio no esta elevada en las usuarias, son factores que debemos tener en cuenta en la decisión costo/beneficio en el uso o no de un anticonceptivo hormonal.

El uso de la AOC de ciclo extendido agrega, aparte de los consabidos beneficios no anticonceptivos de los $A O C$ en general, una muy buena alternativa terapéutica, en los aspectos físicos o psíquicos en la mujer en la cual la necesidad clínica así lo amerita. Criterios relevantes que inciden en la indicación médica para esta forma especial de uso de AOC son las condiciones clínicas de dismenorrea, hipermenorrea acompañada o no de anemia, y la presencia del síndrome de tensión o disfórico premenstrual, sin soslayar, además, en la indicación, los deseos propios de las pacientes de espaciar sus periodos menstruales ya sea por razones sanitarias o comodidad.

Finalmente, esta experiencia nos recuerda y demuestra, que la respuesta a cualquier tipo de fármaco, es personalizada e individual, por lo que la educación de la usuaria actual y futura, en lo que a este punto respecta, es un elemento a considerar en la medicina actual.

\section{BIBLIOGRAFÍA}

1. Bahamondes L, Cordova-Egüez S, Pons JE, et al. Perspectivas sobre el síndrome premenstrual/trastorno disfórico premenstrual. Dis Manage Health Outcomes 2007;15(5):263-77.

2. Anderson FD, Hait H, the Seasonale-301 Study Group. A multicenter, randomized study of an extended cycle oral contraceptive. Contraception 2003;68:9-96.

3. den Tonkellar I, Oddens BJ. Preferred frequency and characteristics of menstrual bleeding in relation to reproductive status, oral contraceptive use, and hormone replacement use. Contraception 1999;59:357-62.

4. Gómez MA. Is menstruation necessary? Spanish women's point of view. Eur J Contracept Reprod Health Care 2008;(Suppl 2):192-3.

5. Fruzzetti F. Contraception without bleeding. Attitude of Italian women. Eur J Contracept Reprod Health Care 2008;(Suppl 2):194.

6. Bonassi Machado R, de Melo NR, Maia H Jr. Bleeding patterns and menstrual-related symptoms with the continuous use of a contraceptive combination of ethinylestradiol and drospirenone: a randomized study. Contraception 2010;81(3):215-22.

7. Kroll R, Reape KZ, Margolis M. The efficacy and safety of a low-dose, 91 day, extended-regimen oral contraceptive with continuous ethinyl estradiol. Contraception 2010;81(1):41-8. 
8. Teichmann A, Apter D, Emerich J, et al. Continuous daily levonorgestrel/ethinyl estradiol vs. 21-day, cyclic levonorgestrel/ethinylestradiol: efficacy, safety and bleeding in a randomized, open-label trial. Contraception 2009; 80(6):504-11.

9. Cremer M, Phan-Weston S, Jacobs A. Recent innovations in oral contraception. Semin Reprod Med 2010; 28(2):140-6.

10. Bonassi Machado R, de Melo NR, Maia H Jr, et al. Effect of a continuous regimen of contraceptive combination of ethinylestradiol and drospirenone on lipid, carbohydrate and coagulation profiles. Contraception 2010;81(2):102-6.

11. Wiegratz I, Stahlberg S, Manthey T, et al. Effects of an oral contraceptive containing $30 \mathrm{mcg}$ ethinyl estradiol and $2 \mathrm{mg}$ dienogest on lipid metabolism during 1 year of conventional or extended-cycle use. Contraception 2010;81(1):57-61.

12. Guazelli CA, Barreiros FA, Barbosa R, et al. Extended regimens of the vaginal contraceptive ring: cycle control. Contraception 2009; 80(5):430-5.

13. Barreiros FA, Guazelli CA, Barbosa R, et al. Extended regimens of the contraceptive vaginal ring: evaluation of clinical aspects. Contraception 2010;81(3):223-5.

14. World Health Organization. Medical Eligibility Criteria for Contraceptive use. Reproductive Health and Research. Fourth Ed. Geneva: WHO, 2009.

15. Lidegaard O. Oral Contraceptives and venous thromboembolism. Dose reduction matters. The 10th Congress of the European Society of Contraception. Prague, Czech Republic, 30th April-3rd May, 2008.

16. Pearlstein TB, Bachman GA, Zacur HA, et al. Treatment of premenstrual dysphoric disorder with a new drospirenone-containing oral contraceptive formulation. Contraception 2005;72(6):414-21.

17. Yonkers KA, Brown C, Pearlstein TB, et al. Efficacy of a new low-dose oral contraceptive with drospirenone in premenstrual dysphoric disorder. Obstet Gynecol 2005;106(3):492-501.

18. Hernádi L, Marr J, Trummer D, et al. Efficacy and safety of a low-dose combined oral contraceptive containing drospirenone $3 \mathrm{mg}$ and ethinylestradiol $20 \mathrm{mcg}$ in a 24/4 day regimen. Contraception 2009;80(1):18-24.

19. Anttila L, Kunz M, Marr J. Bleeding pattern with drospirenone $3 \mathrm{mg}+$ ethinyl estradiol $20 \mathrm{mcg} 24 / 4$ combined oral contraceptive compared with desogestrel 150 mcg+ethinyl estradiol $20 \mathrm{mcg} 21 / 7$ combined oral contraceptives. Contraception 2009;80(5):445-51.

20. Coffee AL, Sulak PJ, Kuehl TJ. Long-term assessment of symptomatology and satisfaction of an extended oral contraceptive regimen. Contraception 2007;75:444-9.

21. Anderson FD, Hait $\mathrm{H}$, Hsiu J, et al. Endometrial microstructure after long-term use of a 91-day extended-cycle oral contraceptive regimen. Contraception 2005; 71:55-9.

22. Mueck AO, Schneidereit R, Heithecker R, et al. Enhancement of the specific advantages of the drospirenone-containing pill by long-cycle contraception. Eur $J$ Contracept Reprod Health Care 2004;(suppl 1):142.

23. Machado RB, Fabrini P, Maia EMC, et al. Bleeding patterns in users of a continuous contraceptive combination containing ethinyl-estradiol and gestodene. Eur $J$ Contracept Reprod Health Care 2004;(suppl 1):133.

24. Machado RB, Fabrini, Padua EMP, et al. Effects of the continuous use of a combination of ethinyl-estradiol/ gestodene in the control of symptoms reported during the hormone-free interval. Eur J Contracept Reprod Health Care 2004;(suppl 1):132.

25. Seidman D, Berr A, Porat N, et al. Prospective followup of an extended regimen using oral contraceptive pills containing ethinyl estradiol and drospirenone. Eur J Contracep Reprod Health Care 2008;(Suppl 2):42.

26. Prilepskaya V, Chernysheva E. Non-contraceptive effects of combined oral contraceptive Marvelon in the prolonged regimen. Eur J Contracep Reprod Health Care 2008;(Suppl 2):122.

27. Mirjana A, Gordana K, Artur B. Acceptance of extended regimens of use of oral hormone contraception in therapeutic purpose. Eur J Contracep Reprod Health Care 2008; (Suppl 2):112.

28. Barreiros F, Guazzelli C, Barbieri M, et al. Bleeding patterns of women using extended regimens of the contraceptive vaginal ring and combined oral contraceptives. Eur J Contracep Health Care 2008;(Suppl 2):110.

29. Guazzelli C, Barreiro F, Araujo F, et al. Extended regimens of the contraceptive vaginal ring and combined oral contraceptives: evaluation of clinical aspects. Eur J Contracep Health Care 2008;(Suppl 2):108.

30. Hannaford PC, Iversen L, Macfarlane TV, et al. Mortality among contraceptive pill users: cohort evidence from Royal College of General Practitioners'Oral contraception study. BMJ 2010;340:c927.

31. Hannaford PC, Selvaraj S, Elliot AM, et al. Cancer risk among oral contraceptive users: cohort data from Royal College of General Practitioner's oral contraception study. BMJ 2007;335:651.

32. Vessey M, Yeates D, Flynn S. Factors affecting mortality in a large cohort study with special reference to oral contraceptive use. Contraception 2010;82:221-9. 